\title{
Defectos congénitos en un hospital de tercer nivel en Cali, Colombia
}

\author{
Julián Ramírez-Cheyne ${ }^{1,2}$, Harry Pachajoa, $P h D^{3}$, Yoseth Ariza, $M S c^{3}$, Carolina Isaza, \\ $M S c^{1}$, Wilmar Saldarriaga, MSc. ${ }^{1,4}$ \\ ${ }^{1}$ Universidad del Valle. ${ }^{2}$ Programa Jóvenes Investigadores e Innovadores "Virginia Gutiérrez de Pineda" de Colciencias, \\ Convocatoria 2010. ${ }^{3}$ Universidad Icesi. ${ }^{4}$ Departamento de Ginecología y Obstetricia, Hospital Universitario del Valle \\ "Evaristo Garcia". Cali, Colombia.
}

\section{RESUMEN}

Objetivo: Determinar la prevalencia global e individual de los defectos congénitos diagnosticados al nacimiento en un hospital de referencia de la ciudad de Cali, Colombia, periodo 2011-2012 y comparar los resultados con lo reportado previamente para la misma institución. Métodos: Se realizó vigilancia epidemiológica de defectos congénitos en el Hospital Universitario del Valle entre julio 2011 y junio 2012, siguiendo la metodología ECLAMC. Se realizaron tablas para ilustrar la distribución de la frecuencia de los defectos congénitos. Resultados: Durante los 12 meses de estudio, se atendieron 5.669 nacimientos, de los cuales 109 presentaron al menos un defecto congénito, para una prevalencia de 1,92\%. Para defectos congénitos específicos se registraron las prevalencias más altas para: polidactilia $(24,69 \times 10.000)$, apéndice preauricular (15,87 x 10.000), hidrocefalia (15,87 x 10.000), hidronefrosis (15,87 x 10.000). Al agruparlos, las prevalencias más altas fueron para los siguientes grupos: defectos de las extremidades $(79,37 \times 10.000)$, defectos del sistema nervioso central (49,39 x 10.000), defectos por disrupción vascular (40,57 x 10.000). Conclusiones: En el periodo de vigilancia en la institución se encontró una prevalencia de defectos congénitos similar a la reportada previamente en la misma. Al agruparlos, las prevalencias más altas fueron para los defectos de las extremidades, defectos del sistema nervioso central y defectos por disrupción vascular. Los defectos congénitos relacionados con factores ambientales, principalmente defectos del tubo neural y defectos por disrupción vascular presentaron prevalencias más altas en comparación con lo reportado previamente.

\section{PALABRAS CLAVE: Anomalías congénitas, epidemiología, cuidado del niño, Colombia}

\section{SUMMARY}

Aims: To determine the overall and individual prevalence of birth defects diagnosed at birth in a referral hospital in Cali, Colombia, in the period 2011-2012 and compare the results with previously reported for the same institution. Methods: Epidemiological surveillance of birth defects was performed at the Hospital Universitario del Valle between July 2011 and June 2012, following ECLAMC methodology. Tables were performed to illustrate the frequency distribution of birth defects. Results: During the 12 months of study there were 5,669 births, of which 109 had at least one birth defect, for a prevalence of 1.92\%. For specific birth defects, the highest prevalence were recorded for polydactyly $(24.69 \times 10,000)$, auricular appendage $(15.87 \times 10,000)$, hydrocephalus $(15.87 \times 10,000)$, hydronephrosis $(15.87 \times 10,000)$. By grouping, the highest prevalence was for the following groups: limb defects $(79.37 \times 10,000)$, central nervous system defects $(49.39 \times 10,000)$, vascular disruption defects $(40.57 \times 10,000)$. Conclusions: In the surveillance period in 
the institution it was found a prevalence of birth defects similar to that previously reported in the same institution. By grouping, the highest prevalence were for limb defects, defects of the central nervous system and vascular disruption defects. Birth defects associated with environmental factors, principally neural tube defects and defects by vascular disruption had higher prevalence compared with those reported previously.

\section{KEYWORDS: Congenital abnormalities, epidemiology, child care, Colombia}

\section{INTRODUCCIÓN}

La organización Mundial de la Salud define los defectos congénitos como todas las anomalías del desarrollo morfológico, estructural, funcional o molecular, presentes al nacer, externas o internas, familiares o esporádicas, hereditarias o no, únicas o múltiples (1). Los defectos congénitos afectan aproximadamente el $3 \%$ de los recién nacidos y causan cerca del $20 \%$ de las muertes en el periodo neonatal $(2,3)$. Desde hace 20 años los defectos congénitos son la principal causa de mortalidad infantil en los Estados Unidos (4) y desde 1994 son la segunda causa de muerte en menores de un año en el Valle del Cauca y Colombia $(5,6)$.

Actualmente se llevan a cabo en el mundo, algunos programas de vigilancia epidemiológica de defectos congénitos. En Europa funcionan el European Surveillance of Congenital Anomalies (EUROCAT) (7) y el Estudio Colaborativo Español de Malformaciones Congénitas (ECEMC) (8). En Latinoamérica existen diferentes registros entre ellos el Registro Cubano de Malformaciones Congénitas (RECUMAC), el Registro de Malformaciones Congénitas en Costa Rica, Vigilancia Epidemiológica de Malformaciones Congénitas Externas (RYVEMCE) en México, y el Estudio Colaborativo Latinoamericano de Malformaciones Congénitas (ECLAMC) que cubre la mayor parte de Sur América $(9)$.

En 1967 se inició el ECLAMC, que en diferentes épocas ha incluido Colombia $(10,11)$, y desde el año 2001 viene funcionando de manera ininterrumpida, inicialmente en la ciudad de Bogotá (5), luego en Manizales y Ubaté y desde marzo del 2004, en Cali con el Hospital Universitario del Valle (HUV) (12).

Durante los años de funcionamiento del ECLA$\mathrm{MC}$ en Cali se han identificado patrones y agregaciones de la ocurrencia de defectos congénitos en la ciudad y se están llevando a cabo estudios complementarios para la evaluación de posibles factores de riesgo $(13,14)$.

El presente trabajo tiene como objetivo describir la ocurrencia de los defectos congénitos diagnosticados al nacimiento de los recién nacidos afectados en un hospital de tercer nivel de la ciudad de
Cali, Colombia, durante el período de julio de 2011 a junio de 2012 .

\section{MATERIALES Y MÉTODOS}

Los datos fueron recolectados en el HUV en la ciudad de Cali. EI HUV es el mayor centro de referencia de la red pública de Cali y es el hospital que atiende el mayor número de partos en la ciudad. Debido al alto nivel de complejidad de los servicios que presta este hospital, a él se remiten la mayoría de las mujeres embarazadas con patologías que necesitan un nivel III y IV de atención, incluidas aquellas con diagnóstico prenatal de defectos congénitos. El área de influencia del HUV incluye principalmente el departamento del Valle del Cauca que según datos del Departamento Administrativo Nacional de Estadística (DANE) con datos del censo del 2005 sumaba una población de 4.052.535 habitantes (15).

La recolección de la información de los casos fue realizada por el Joven Investigador, médico encargado de evaluar diariamente todos los recién nacidos intrahospitalarios, de acuerdo a la descripción recomendada por el manual operacional del ECLAMC versión 2002 (9). De acuerdo con ese manual, se definió como caso a todo RN vivo o mortinato con peso mayor a 500 gramos cuyo parto fuera atendido en el HUV, con presencia de uno o más defectos congénitos mayores detectado por examen físico, durante las primeras 24 horas después del nacimiento.

Análisis estadístico: El análisis exploratorio de los datos se realizó mediante la construcción de tablas resumen con la distribución según el tipo de defectos. Posteriormente, se calcularon medidas de ocurrencia de los defectos congénitos individuales y agrupados. Las tasas de prevalencia se expresan por 10.000 nacimientos.

\section{RESULTADOS}

Durante el período de estudio de 12 meses entre julio de 2011 y junio de 2012, se atendieron 5.669 nacimientos. De ellos, 109 presentaron al menos un defecto congénito que se diagnosticó al nacimiento, para una prevalencia de $1,92 \%$. 
Entre los casos registrados, 98 (89,9\%) nacieron vivos, $11(10,1 \%)$ nacieron muertos, 58 $(53,21 \%)$ de sexo masculino, 49 (44,95\%) de sexo femenino, y en $2(1,83 \%)$ no se definió el sexo, 67 $(61,47 \%)$ procedían de la ciudad de Cali y los 42 restantes $(38,53 \%)$ de los municipios aledaños y otros departamentos.
En la Tabla I se muestran las prevalencias individuales estimadas de los defectos congénitos registrados. Los defectos congénitos con prevalencias más altas fueron: polidactilia $(24,69 \times 10.000)$, apéndice preauricular $(15,87 \times 10.000)$, hidrocefalia $(15,87 \times 10.000)$, hidronefrosis $(15,87 \times 10.000)$, cardiopatía $(14,11$ x 10.000) y pie equinovaro $(14,11 \times 10.000)$.

Tabla I

PREVALENCIAS INSTITUCIONALES DE LOS DEFECTOS CONGÉNITOS AISLADOS

\begin{tabular}{|c|c|c|c|}
\hline Defecto congénito & $\begin{array}{c}\text { Prevalencia x } \\
10.000 \\
(1987-1988)\end{array}$ & $\begin{array}{c}\text { Prevalencia x } \\
10.000 \\
(2004-2008)\end{array}$ & $\begin{array}{c}\text { Prevalencia } x \\
10.000 \\
(2011-2012)^{\star}\end{array}$ \\
\hline Polidactilia & 34,2 & 22,12 & $14 / 24,69$ \\
\hline Hidrocefalia & 4,2 & 16,37 & $9 / 15,87$ \\
\hline Hidronefrosis & $\ldots$ & 11,52 & $9 / 15,87$ \\
\hline Apéndices preauriculares & $\cdots$ & $\ldots$ & $9 / 15,87$ \\
\hline Cardiopatía & 4,2 & 14,65 & $8 / 14,11$ \\
\hline Pie equinovaro & 8,5 & 17,58 & $8 / 14,11$ \\
\hline Espina bífida & $\ldots$ & 7,27 & $7 / 12,34$ \\
\hline Síndrome de Down & $\ldots$ & 9,4 & $6 / 10,58$ \\
\hline Agenesia renal & $\cdots$ & 1,52 & $5 / 8,81$ \\
\hline Encefalocele & $\cdots$ & 3,03 & $5 / 8,81$ \\
\hline Gastrosquisis & $\cdots$ & 7,27 & $5 / 8,81$ \\
\hline Labio y paladar hendido & 8,5 & 10,91 & $5 / 8,81$ \\
\hline Sindactilia & 2,1 & 4,55 & $5 / 8,81$ \\
\hline Anomalías del pulgar & $\ldots$ & $\ldots$ & $5 / 8,81$ \\
\hline Secuencia de Moebius & $\cdots$ & $\cdots$ & $4 / 7,05$ \\
\hline Atresia esofágica & $\cdots$ & 3,03 & $3 / 5,29$ \\
\hline Secuencia de Pierre-Robin & $\ldots$ & $\ldots$ & $3 / 5,29$ \\
\hline Luxación de rodilla & $\ldots$ & $\ldots$ & $3 / 5,29$ \\
\hline Síndrome de Turner & $\ldots$ & $\ldots$ & $3 / 5,29$ \\
\hline Hipoplasia/defectos longitudinales de las extremidades superiores & $\ldots$ & $\ldots$ & $3 / 5,29$ \\
\hline Anencefalia & $\cdots$ & 6,36 & $2 / 3,52$ \\
\hline Atresia anal/rectal & 1 & 6,06 & $2 / 3,52$ \\
\hline Defectos transversales de las extremidades & $\ldots$ & $\ldots$ & $2 / 3,52$ \\
\hline Luxación de cadera & 36,4 & 2,72 & $2 / 3,52$ \\
\hline Microcefalia & $\ldots$ & 1,52 & $2 / 3,52$ \\
\hline Onfalocele & $\cdots$ & 3,64 & $2 / 3,52$ \\
\hline Riñón poliquístico & $\ldots$ & 1,52 & $2 / 3,52$ \\
\hline Mal rotación intestinal & $\ldots$ & $\ldots$ & $2 / 3,52$ \\
\hline Arteria umbilical única & $\ldots$ & $\cdots$ & $2 / 3,52$ \\
\hline Aplasia cutis & $\ldots$ & $\cdots$ & $2 / 3,52$ \\
\hline Hidranencefalia & $\cdots$ & $\cdots$ & $2 / 3,52$ \\
\hline Hipoplasia/defectos longitudinales de las extremidades inferiores & $\cdots$ & $\cdots$ & $2 / 3,52$ \\
\hline Macrocórnea & $\cdots$ & $\cdots$ & $2 / 3,52$ \\
\hline Ambigüedad sexual & 3,2 & 2,12 & $2 / 3,52$ \\
\hline
\end{tabular}


CONTINUACIÓN TABLA I

\begin{tabular}{|c|c|c|c|}
\hline Defecto congénito & $\begin{array}{c}\text { Prevalencia x } \\
10.000 \\
(1987-1988)\end{array}$ & $\begin{array}{c}\text { Prevalencia x } \\
10.000 \\
(2004-2008)\end{array}$ & $\begin{array}{c}\text { Prevalencia x } \\
10.000 \\
(2011-2012)^{\star}\end{array}$ \\
\hline Artrogriposis múltiple congénita & $\cdots$ & 0,61 & $1 / 1,76$ \\
\hline Atresia duodenal & $\cdots$ & 2,12 & $1 / 1,76$ \\
\hline Atresia de yeyuno-íleon & $\ldots$ & 0,61 & $1 / 1,76$ \\
\hline Atresia de intestino grueso & $\cdots$ & $\ldots$ & $1 / 1,76$ \\
\hline Hipospadias & 5,3 & 6,06 & $1 / 1,76$ \\
\hline Microtia & $\cdots$ & 3,03 & $1 / 1,76$ \\
\hline Macrocránea & $\cdots$ & $\cdots$ & $1 / 1,76$ \\
\hline Anquiloglosia & $\cdots$ & $\cdots$ & $1 / 1,76$ \\
\hline Albinismo & $\cdots$ & $\cdots$ & $1 / 1,76$ \\
\hline Macrostomía & $\ldots$ & $\cdots$ & $1 / 1,76$ \\
\hline Espectro oculoauriculovertebral & $\cdots$ & $\cdots$ & $1 / 1,76$ \\
\hline Esquizencefalia & $\cdots$ & $\cdots$ & $1 / 1,76$ \\
\hline Quiste pulmonar & $\cdots$ & $\cdots$ & $1 / 1,76$ \\
\hline Piebaldismo & $\cdots$ & $\cdots$ & $1 / 1,76$ \\
\hline Situs inversus & $\cdots$ & $\ldots$ & $1 / 1,76$ \\
\hline Pterigium múltiple & $\cdots$ & $\cdots$ & $1 / 1,76$ \\
\hline Microstomía & $\cdots$ & $\cdots$ & $1 / 1,76$ \\
\hline Síndrome de Klinefelter & $\cdots$ & $\cdots$ & $1 / 1,76$ \\
\hline Ectopia renal & $\cdots$ & $\cdots$ & $1 / 1,76$ \\
\hline Vértebra en cuña & $\cdots$ & $\cdots$ & $1 / 1,76$ \\
\hline Abdomen en uva pasa & $\cdots$ & $\cdots$ & $1 / 1,76$ \\
\hline Ciclopía & $\cdots$ & 2,42 & 0 \\
\hline Sirenomelia & 1 & 1,21 & 0 \\
\hline
\end{tabular}

${ }^{\star}$ Casos/Prevalencia del actual estudio. Prevalencia x 10.000 nacimientos.

Sin embargo, al agrupar los defectos (Tabla II) las prevalencias más altas fueron para los siguientes grupos: defectos de las extremidades $(79,37$ $x$ 10.000), defectos del sistema nervioso central $(49,39 \times 10.000)$ y defectos por disrupción vascular $(42,33 \times 10.000)$.

\section{DISCUSIÓN}

La prevalencia de los defectos congénitos en este hospital de Cali, durante el periodo de julio de 2011 a junio de 2012 fue de 1,92\%. En Colombia, la prevalencia de los defectos congénitos ha sido estimada en diferentes ciudades en niveles similares entre $2,2 \%$ y $3,20 \%$, como se indica en la Tabla III (16-22). En 1989 la prevalencia de defectos congénitos en este mismo hospital fue estimada en $2,3 \%$ (17), y en el periodo de 2004 a 2008 se obtuvo $2,22 \%$ (22), lo que sugiere que la ocurrencia total de defectos ha permanecido estable alrededor de $2 \%$ en los últimos años.

En el presente estudio, el $53,21 \%$ de los recién nacidos con defectos congénitos era de sexo masculino con una relación hombre/mujer de 1,18 lo que coincide con lo reportado en la literatura mundial y con estudios recientes en la misma institución $(23,22)$.

Aunque un poco más alta, se repite con respecto a estudios recientes en la misma institución, una baja prevalencia de la luxación de cadera, que de acuerdo a registros previos, pasó de una prevalencia de 36,4 × 10.000 en 1988 a 2,7 por 10.000 nacimientos durante el periodo de 2004 a 2008, y en este estudio fue de $3,52 \times 10.000$, lo cual puede estar indicando un subdiagnóstico. 


\section{PREVALENCIAS INSTITUCIONALES DE DEFECTOS CONGÉNITOS AGRUPADOS}

\begin{tabular}{|c|c|c|c|}
\hline Grupos defectos congénitos & $\begin{array}{l}\text { Prevalencia } \\
\text { x 10.000 } \\
(2004-008)\end{array}$ & $\begin{array}{c}\text { Prevalencia } \\
\text { x 10.000 } \\
(2011-2012)^{\star}\end{array}$ & $\begin{array}{l}\text { Defectos individuales incluidos en } \\
\text { cada grupo }\end{array}$ \\
\hline
\end{tabular}

Defectos de las extremidades $\quad 55,76 \quad 45 / 79,37$

dactilia, pie equinovaro, anomalías del pulgar, sindactilia, hipoplasia/defectos longitudinales de las extremidades superiores, luxación de rodilla, hipoplasia/defectos longitudinales de las extremidades inferiores, luxación de cadera, defectos transversales de las extremidades, artrogriposis múltiple congénita

Defectos del sistema nervioso central

36,97

25,76

Defectos por disrupción vascular

Defectos genitourinarios

Defectos del tubo neural

Anomalías del tracto gastrointestinal
16,36

11,82
$24 / 42,33$

$28 / 49,39$

Hidrocefalia, espina bífida, encefalocele, anencefalia, hidranencefalia, microcefalia, esquizencefalia, ciclopía

Agenesia renal, gastrosquisis, secuencia de Moebius, aplasia cutis, atresia anal, defectos transversales de las extremidades, hidranencefalia, atresia intestinal, espectro oculoauriculovertebral

Hidronefrosis, agenesia renal, riñón poliquístico, abdomen en uva pasa, ambigüedad sexual, ectopia renal, hipospadias

Espina bífida, encefalocele, anencefalia

Atresia esofágica, atresia intestinal, atresia anal, malrotación intestinal

${ }^{*}$ Casos/Prevalencia del actual estudio. Prevalencia x 10.000 nacimientos.

Tabla III

PREVALENCIA DE DEFECTOS CONGÉNITOS SEGÚN AUTOR Y AÑO DE PUBLICACIÓN REALIZADOS EN COLOMBIA

\begin{tabular}{lccc}
\hline Autor, año & Ciudad de estudio & Nacimientos & Prevalencia (\%) \\
\hline Silva, 1984 & Cartagena & 6.805 & 3,2 \\
Isaza, 1989 & Cali & 9.103 & 2,3 \\
Pinto,1990 & Barranquilla & 8.469 & 2,2 \\
Giraldo,1992 & Bogotá & 7.752 & 2,7 \\
Arteaga, 1993 & Bogotá & 9.224 & 2,7 \\
Giraldo, 2003 & Bogotá & 5.686 & 3,0 \\
Fernández, 2007 & Bogotá & 54.397 & 3,0 \\
Pachajoa, 2010 & Cali & 32.995 & 2,2 \\
Presente estudio & Cali & 5.669 & 1,92 \\
\hline
\end{tabular}


Los defectos del tubo neural (DTN), desde 1988 hasta el periodo 2004-2008, habían disminuido la prevalencia de 31 x 10.000 a 16,66 x 10.000 nacimientos $(12,22)$, los autores atribuyeron esto a la fortificación de la harina de trigo, con ácido fólico, que viene realizándose en Colombia desde 1996 (24). Antes del estudio de Pachajoa y cols (22), esta disminución no había sido reportada en Colombia, principalmente porque la mayoría de los registros de defectos congénitos son posteriores al inicio de la fortificación.

Similares hallazgos ya habían sido reportados en Chile, donde se inició un programa de fortificación de la harina de trigo con ácido fólico con el objetivo de disminuir la frecuencia de DTN $(25,26)$. En la población chilena en el año 2005 LópezCamelo y cols (27), reportaron una disminución de aproximadamente un $51 \%$ para espina bífida (mínimo $27 \%$, máximo $66 \%$ ), y de $46 \%$ para anencefalia (mínimo 15\%, máximo 66\%). En el presente estudio, se registraron 7 casos de espina bífida, 5 casos de encefalocefe y 2 casos de anencefalia, es decir que para los DTN en conjunto hubo 14 casos y una prevalencia de $24,69 \times 10.000$, esta prevalencia, no es tan alta como la reportada para el HUV en 1987-1988, ni tan baja como la reportada para tal institución en 2004-2008, y sería recomendable evaluar los niveles de ácido fólico directamente en las mujeres en edad fértil para evaluar los efectos de la fortificación.

Los sistemas de vigilancia de malformaciones congénitas permiten monitorear cambios en la prevalencia de los defectos congénitos y detectar patrones inusuales de agregación temporal y/o espacial que puedan sugerir la influencia de factores medioambientales o de exposición individual. Nuestro registro ha permitido detectar dos conglomerados ("clusters") de defectos congénitos de baja frecuencia para sirenomelia y ciclopía, lo que dio lugar a plantear una hipótesis sobre el origen de los conglomerados y evaluar exposición a factores medioambientales $(13,14)$.

Ciertos defectos congénitos han mostrado prevalencias aumentadas en estudios recientes en la institución, como la atresia anal $(6,06 \times 10.000)$, la ciclopía $(2,42 \times 10.000)$ y la sirenomelia $(1,21 \times$ $10.000)$, y se ha sugerido que pudieran estar asociados a la exposición a contaminantes y teratógenos (22). En el presente estudio no se encontraron casos de ciclopía ni sirenomelia y se hubo una menor prevalencia $(3,52 \times 10.000)$ para la atresia anal.

En el estudio realizado entre 2004 y 2008, llamó la atención que, en comparación con el ECLAMC, en el registro del HUV en Cali, la prevalencia de los casos de defectos por disrupción vascular (DDV) estaba aumentada (22). Estos defectos son alteraciones estructurales del desarrollo producidas por problemas vasculares como vasoconstricción severa intermitente, regresión anormal de vasos du- rante la remodelación del sistema vascular, trombosis arterial o cualquier fenómeno que produzca falta de $\mathrm{O} 2$ en un tejido u órgano, durante periodos específicos del desarrollo, y que conllevan a destrucción del tejido o detención de su desarrollo (28). Se ha sugerido que puedan presentarse por agregaciones temporales o espaciales (29-31). Se han considerado como DDV a los defectos transversos de las extremidades, atresias intestinales, gastrosquisis, hidranencefalia, porencefalia, secuencia de hipogénesis oromandibular y de las extremidades, espectro oculoauriculovertebral, agenesia renal, aplasia cutis y síndrome de Moebius (32-45).

En Colombia existen estudios que muestran disparidad en el acceso oportuno a un control prenatal de calidad e inequidad en salud maternoinfantil $(46,47)$. El HUV, es un hospital de referencia de la red pública que atiende una población materna de bajos recursos económicos, bajo nivel de educación y sometida a la inequidad mencionada, lo que sumado a un predominio importante de mujeres jóvenes y primigestantes, podría estar influenciando la ocurrencia de DDV. También se han asociado a la ocurrencia de este tipo de defectos, el cigarrillo y, en el caso específico de Cali, el relleno sanitario de Navarro (botadero municipal de residuos domésticos que operó en Cali entre 1967 y 2008) $(13,14,22)$. Con respecto a lo encontrado en el periodo entre 2004 y 2008 en el HUV, en el presente estudio se encontró que, aumentaron las prevalencias de la agenesia renal $(1,52 \times 10.000$ a $8,81 \times$ $10.000)$ y las atresias intestinales $(2,73 \times 10.000$ a $5,28 \times 10.000$ ), y se mantuvo la prevalencia de gastrosquisis $(7,27 \times 10.000$ a $8,81 \times 10.000)$, las prevalencias individuales del resto de DDV se pueden observar en la Tabla I. Si se agrupan todos los DDV registrados en el estudio entre 2004 y 2008, se obtiene una prevalencia de 25,76 x 10.000, en el presente estudio se registraron 24 casos, lo que implica una prevalencia conjunta de 42,33 x 10.000 para todos los DDV. Se requieren estudios específicos sobre los DDV, enfocados en la identificación de factores de riesgo para su ocurrencia.

\section{CONCLUSIÓN}

Durante el período de estudio de 12 meses entre julio de 2011 y junio de 2012 en la institución del estudio se determinó una prevalencia de defectos congénitos similar a lo reportado anteriormente en la misma. Los defectos congénitos aislados con prevalencias más altas fueron: polidactilia, apéndice preauricular e hidrocefalia. Al agrupar los defectos congénitos, las prevalencias más altas fueron para: defectos de las extremidades, defectos del sistema nervioso central y defectos por disrupción vascular. Los defectos congénitos relacionados con factores ambientales, principalmente defectos del tubo neural y defectos por disrupción vascular 
presentaron prevalencias más altas en comparación con lo reportado previamente.

Agradecimientos: A los miembros del ECLAMC del HUV. Este proyecto fue financiado por Colciencias y la Universidad del Valle, en el marco del Programa Jóvenes Investigadores e Innovadores "Virginia Gutiérrez de Pineda" de Colciencias. Convocatoria 2010.

\section{REFERENCIAS}

1. Castilla E, Lopez J, Paz J, Orioli E. Prevención primaria de los defectos congénitos. Rio de Janeiro: Fio Cruz; 1996.

2. D'Alton ME, DeCherney $\mathrm{AH}$. Prenatal diagnosis. N Engl J Med 1993;328:114-20.

3. Yoon PW, Olney RS, Khoury MJ, Sappenfield WM, Chavez GF, Taylor D. Contributions of birth defects and genetic diseases to pediatric hospitalizations: a population-based study. Arch Pediatr Adolesc Med 1997;151(11):1096-103.

4. Petrini J, Damus K, Russell R, Poschman K, Davidoff MJ, Mattison D. Contribution of birth defects to infant mortality in the United States. Teratology 2002; 66(Suppl 1):S3-S6.

5. Fernández N, Zarante I. Prevalencia y escala pronóstico para malformaciones congénitas en Colombia: La responsabilidad de pediatras y neonatólogos. Registro de 54.397 nacimientos. UCIN 2007;7(4):28-32.

6. Instituto Geográfico Agustín Codazzi. Ministerio de Protección Social. Atlas de Salud. 1를 edición. Bogotá: Imprenta Nacional de Colombia; 2008.

7. Eurocat Working Group: Surveillance of Congenital Anomalies 1980-1988, Eurocat Report No.4: BruxeIles Department of Epidemiology, Catholic University of Louvain; 1991.

8. Bermejo E, Cuevas L, Mendioroz J, Martínez Frías ML. Vigilancia epidemiológica de anomalías congénitas en España, en los últimos 24 años. Boletín del Ecemc: Revista de Dismorfología y Epidemiología 2004;5:57-82.

9. Castilla EE, Orioli I. ECLAMC: The Latin-American Collaborative Study of Congenital Malformations. Community Genet 2004;7:76-94.

10. Castilla EE, Orioli IM. Estudio colaborativo latinoamericano de anomalías congénitas: ECLAMC/MONITOR. Interciencia 1983;3:271-8.

11. López-Camelo SJ, Orioli IM. Heterogeneus rates for birth defects in Latin America: hints of causality. Genet Epidemiol 1996;13:469-81.

12. Pachajoa $H$, Saldarriaga W, Isaza $C$. Gastrosquisis en un hospital de tercer nivel de la ciudad de Cali, Colombia durante el período marzo 2004 a febrero 2006. Colomb Med 2008;39(s2):35-40.

13. Castilla EE, Mastroiacovo P, López-Camelo JS, Saldarriaga W, Isaza C, Orioli IM. Sirenomelia and Cyclopia Cluster in Cali, Colombia. Am J Med Genetics. Part A. 2008;146A:2626-36.

14. Orioli IM, Mastroiacovo P, López-Camelo JS, Saldarriaga $\mathrm{W}$, Isaza $\mathrm{C}$, Aiello $\mathrm{H}$, et al. Clusters of sirenomelia in South America. Birth Defects Res A Clin Mol Teratol 2009;85(2):112-8.
15. Departamento Administrativo Nacional de Estadística-DANE. Internet Censo básico 2005. Dane. Disponible en: http://www.dane.gov.co

16. Silva JR. Evaluación genética y estudio de malformaciones congénitas. Acta Pediátrica Colombiana 1984;2:12-23.

17. Isaza C, Martina D, Estupiñán J, Stark C, Rey H. Prevalencia de anomalías congénitas diagnosticadas en las primeras 24 horas de vida. Colombia Médica 1989;20:156-9.

18. Pinto P. Estudio de anomalías congénitas en una población de nacidos en el Instituto de Seguros Sociales (ISS) de Barranquilla, de 1985-1988. Salud Uninorte 1990;3:123-30.

19. Giraldo A. A case control study of clinically detected congenital anomalies in newborn babies and common risk factors (thesis). Baltimore: Johns Hopkins University; 1992.

20. Arteaga CE. Prevalencia y factores asociados a las anomalías congénitas y enfermedades de instalación prenatal en el Instituto Materno Infantil de Bogotá tesis de maestría. Bogotá: Universidad Nacional de Colombia; 1993.

21. Fernández N, Zarante I. Prevalencia y escala pronóstico para malformaciones congénitas en Colombia: La responsabilidad de pediatras y neonatólogos. Registro de 54.397 nacimientos. UCIN 2007;7(4):28-32.

22. Pachajoa H, Ariza $Y$, Isaza $C$, Méndez F. Defectos congénitos mayores en un hospital de tercer nivel en Cali, Colombia2004-2008. Rev Salud Pública. 2010;13(1):152-62.

23. Lisi A, Botto L, Rittler M, Castilla E, Bianca S, Bianchi $F$, et al. Sex and congenital malformations: an international perspective. Am J Med Genet A. 2005;134A:4957.

24. Decreto por el cual se reglamenta la fortificación de la harina de trigo y se establecen las condiciones de comercialización, rotulado, vigilancia y control. Decreto 1944 de 1996. Diario Oficial No. 42.909, de 30 de octubre de 1996.

25. Nazer J, López Camelo J, Castilla EE. Estudio de 30 años de vigilancia epidemiológica de defectos de tubo neural en Chile y en Latino América. Rev Méd Chile 2001;129:531-9.

26. Castilla EE, Orioli IM, López-Camelo J, Graca Dutra M, Nazer J. Preliminary data on changes in Neural Tubes Prevalence rates after folic acid fortification in South America. Am J Med Genet 2003;123A:123-8.

27. López-Camelo, Orioli M, Graca DM, Nazer J, Rivera $\mathrm{N}$, Ojeda ME, et al. Reduction of birth prevalence rates of neural tubes defects after folic acid fortification in Chile. Am J Med Genet 2005;135A:120-5.

28. Webster WS, Abela D. The effect of hypoxia in development. BirthDefects research (Part C). 2007;81:21528.

29. Werler MM, Sheehan JE, Mitchell AA. Maternal medication use and risks of gastroschisis and small intestinal atresia. Am J Epidemiol 2002;155(1):26-31.

30. Werler MM. Teratogen Update: Pseudoephedrine. Birth Defects Res A Clin Mol Teratol 2006;76:445-52.

31. Cuartas DE, Ariza Y, Pachajoa H, Méndez F. Analysis of the spatial and temporal distribution of birth defects between 2004-2008 at a third-level hospital in Cali, Colombia. Colomb Med 2011;42:9-16. 
32. Hoyme HE, Higginbottom MC, Jones KL. The vascular pathogenesis of gastroschisis: Intrauterine interruption of the omphalomesenteric artery. J Pediatr 1981a;98:228-31.

33. Hoyme HE, Higginbottom MC, Jones KL. Vascular etiology of disruptive structural defects in monozygotic twins. Pediatrics 1981b;67:288-91.

34. Hoyme HE, Jones KL, Van Allen MI, Saunders BS, Benirschke K. Vascular pathogenesis of transverse limb reduction defects. J Pediatr 1982;101:839-43.

35. Hoyme HE, Van Allen MI, Jones KL. The vascular pathogenesis of some sporadically occurring limb defects. Semin Perinatol 1983a;7:299-306.

36. Hoyme HE, Jones MC, Jones KL. Gastroschisis: Abdominal wall disruption secondary to early gestational interruption of the omphalo-mesenteric artery. Semin Perinatol 1983b;7:294-8.

37. Louw JH, Barnard CN. Congenital intestinal atresia,observations on its origin. Lancet 1955;269:1065-7.

38. Nayci A, Avlan D, Polat A, Aksoyek S. lleal atresia associated with a congenital vascular band anomaly: Observations on pathogenesis. Pediatr Surg Int 2003;19:742-3.

39. Schinzel AA, Smith DW, Miller JR. Monozygotic twinning and structural defects. J Pediatr 1979;95:921-30.

40. Jung JH, Graham JM Jr, Schultz N, Smith DW. Congenital hydranencephaly/porencephaly due to vascular disruption in monozygotic twins. Pediatrics 1984;73:467-9.
41. Dominguez R, Aguirre Vila-Coro A, Slopis JM, Bohan TP. Brain and ocular abnormalities in infants with in utero exposure to cocaine and other street drugs. Am J DisChild 1991;145:688-95.

42. van der Knaap MS, Smit LM, Barkhof F, Pijnenburg YA, Zweegman S, Niessen HW, Imhof S, Heutink P. Neonatal porencephaly and adult stroke related to mutations in collagen IV A1. Ann Neurol 2006;59:50411.

43. Husain T, Langlois PH, Sever LE, Gambello MJ. Descriptive epidemiologic features shared by birth defects thought to be related to vascular disruption in Texas, 1996-2002. Birth Defects Res A Clin Mol Teratol 2008;82:435-40.

44. van der Zee DC, Bax KM, Vermeij-Keers C. Materno embryonic transfusion and congenital malformations. Prenat Diagn 1997;17:59-69.

45. Poswillo D. The pathogenesis of the first and second branchial arch syndrome. Oral Surg Oral Med Oral Pathol 1973;35:302-28.

46. Saldarriaga GW, Ruíz Murcia FA, Fandiño-Losada A, Cruz Perea ME, Isaza de Lourido C. Evaluation of prenatal diagnosis of congenital anomalies diagnosable by prenatal ultrasound in patients in neonatal intensive care units of Cali, Colombia Colomb Med 2014;45(1):32-8.

47. Ruiz-Murcia FA, Fandiño-Losada A, Ramírez-Cheyne $\mathrm{J}$, Isaza C, Saldarriaga W. Inequidades en el diagnóstico de anomalías congénitas mayores en recién nacidos en Cali, Colombia. Rev Chil Obstet Ginecol 2014;79(6):481-8. 